\title{
ENTREVISTA
}

\section{TEUN A. VAN DIJK Y LOS ESTUDIOS CRÍTICOS DEL DISCURSO (ECD)}

\section{Oscar Iván Londoño Zapata}

Son muchos los investigadores y investigadoras académicas que han escrito y hablado sobre Teun A. van Dijk. Múltiples textos como libros, conferencias, seminarios y ensayos, han dedicado páginas enteras para exponer, analizar, aplicar y valorar las aportaciones teoréticas de este reconocido analista del discurso. Estudios e investigaciones sobre la teoría literaria, la poética generativa, el texto y la gramática textual, la psicología del procesamiento del discurso, el contexto y la pragmática del discurso, el análisis del discurso, el análisis crítico del discurso, las noticias, el racismo, el sexismo, la dominación y el poder, la fijación cognitiva de las creencias, la ideología, el discurso y el conocimiento, entre otros, han sido los campos de acción investigativa de Teun, y han permitido, de igual manera, que se establezca una evolución pertinente en los estudios de la lengua.

Entre sus múltiples publicaciones traducidas en lengua castellana, que desarrollan los campos investigativos anteriormente mencionados, se encuentran: Texto y contexto. Madrid: Cátedra, 1980. La ciencia del texto. Barcelona/Buenos Aires: Paidós, 1983. Las estructuras y funciones del discurso. México: Siglo XXI, 1980. (14a edición actualizada, 2005). La noticia como discurso. Comprensión, estructura y producción de la información. Barcelona, Paidós, 1990. Prensa, racismo y poder. México: Universidad Ibero Americana, 1995. Racismo y análisis crítico de los medios. Barcelona: Paidós, 1997. Ideología. Una aproximación multidisciplinaria. Barcelona/Buenos Aires: Gedisa, 1999. 8. (Ed.). Estudios del discurso. 2 vols. Barcelona/ Buenos Aires: Gedisa, 2000. De la poética generativa hasta el análisis crítico del discurso. Artículos seleccionados 1976-1998. Ámsterdam: Universidad de Ámsterdam. Enero 1999. (Con Iván Rodrigo M.) Análisis del discurso social y político. Quito, Ecuador: Abya-Yala, 1999. Ideología y discurso. Una introducción multidisciplinaria. Barcelona: Ariel, 2003. Racismo y discurso de las élites. Barcelona: Gedisa, 2003. Dominación étnica y racismo discursivo en España y América Latina. Barcelona: Gedisa, 2003. (Ed.) Racismo y discurso en América Latina. Barcelona: Gedisa, 2007. En preparación: 
Discurso y Poder. Barcelona: Gedisa, 2008. Contexto y Discurso. Una aproximación cognitiva. Barcelona: Gedisa, 2008. La Sociedad en el Discurso: Cómo el contexto controla el texto y el habla. Barcelona: Gedisa, 2008.

Es por ello que Teun A. van Dijk se ha posicionado como uno de los académicos e investigadores más importantes en el ámbito de los estudios del discurso a nivel mundial.

OSCAR IVÁN LONDOÑO ZAPATA: Teun recibe un cordial saludo. Es un gusto poder encontrarme contigo otra vez en una nueva entrevista. En septiembre de 2007 se llevó a cabo en Bogotá-Colombia el VII Congreso Latinoamericano de Estudios del Discurso, evento en el que se dieron cita importantes investigadores(as) nacionales e internacionales, presentando sus últimos estudios y hallazgos, ¿Cómo evalúas los adelantos y desarrollos investigativos expuestos?

TEUN A. VAN DIJK: Pese a que estuve pocos días y escuché sólo algunas conferencias puedo concluir que, otra vez, la Asociación Latinoamericana de Estudios del Discurso (ALED), reúne investigadores(as) de gran calidad, y de muchos países, trabajando sobre una variedad impresionante de temas. Uno de los avances más destacables es que, comparado con hace más de diez años, la dependencia, así como la referencia, casi exclusiva de los trabajos norteamericanos y europeos se ha transformado; ahora la investigación en los países de América Latina se destaca por una gran influencia mutua. Después de tantos congresos de la $A L E D$ las personas se conocen, muchas veces como amigos y amigas, y hacen referencia a los trabajos de los demás e incluso desarrollan juntos(as) proyectos de investigación internacionales en América Latina.

O.I.L.Z.: En este congreso presentaste ante la comunidad académica el libro "Racismo y discurso en América Latina”, del cual eres Editor. ¿Podrías realizar por favor un comentario sobre este trabajo multidisciplinario?

T.A.v.D.: Este libro fue el resultado de un proyecto latinoamericano mayor, en el que cooperaron investigadores(as) de ocho países: México, Guatemala, Colombia, Venezuela, Brasil, Argentina, Chile y Perú, con equipos de expertos de varias disciplinas, coordinados(as) por especialistas en racismo y discurso. Después de la publicación de mi libro, muy modesto, Dominación étnica y racismo discursivo en España y América Latina (2003), en el que solamente había una parte sobre América Latina, tenía la convicción que tal trabajo debía hacerse con expertos(as) de los propios países. El objetivo principal de 
este libro fue reunir informes sobre las diferentes formas del racismo en los discursos políticos, así como los de los medios de comunicación, los libros de texto y otros discursos dominantes. Después de varios años de investigación el libro resultó ser un compendio imprescindible de estudios sobre las formas del racismo discursivo en América Latina. Por el momento se publicó una traducción en Brasil en 2007, y una versión inglesa está ya en preparación, y será publicada en Estadios Unidos. También, las coordinadoras y el coordinador de los capítulos individuales están convencidos que cada país necesita un libro monográfico, y no solamente un capítulo. Seguimos en contacto para estos trabajos futuros.

O.I.L.Z.: Muchas de las aplicaciones del Análisis del Discurso (AD) y el Análisis Crítico del Discurso (ACD) desarrolladas por investigadores(as) latinoamericanos(as) tienen como referente teorético y metodológico inmediato enfoques o modelos del AD y el ACD eurocéntricos (Ver: Fairclough y Wodak, 2000). Desde tu perspectiva, lo anterior posibilita o imposibilita la generación de un constructo epistémico propio, que aporte en la descripción e interpretación de los problemas sociales locales latinoamericanos, ¿Cómo entender ésto que alguns han denominado violencia epistémica?

T.A.v.D.: Es cierto que todavía hay muchas referencias a trabajos europeos, pero esto también ocurre en el mundo entero, y no solamente en América Latina, porque el ACD ha sido sobre todo un movimiento europeo - mucho menos en EEUU, donde la investigación crítica, especialmente después el 11 de septiembre de 2001, es mucho menos activa. Pero como acabo de decir, hay una fuerte tendencia de autosuficiencia científica en América Latina. La mayoría de las investigaciones no son de $\mathrm{ACD}$, sino de otras áreas, como el análisis de la conversación, la argumentación, la educación, entre otras. En mis escritos y en mis prácticas como editor de varias revistas recomiendo a las personas no seguir una orientación particular de ACD, sino explorar sus propios caminos. Incluso a mis propios estudiantes de doctorado les pido que no me citen, sólo cuando sea necesario, es decir, cuando aborden directamente los tópicos que he trabajado, como el racismo, la ideología o el contexto. Además, hay tantas líneas interesantes en los estudios del discurso del mundo entero que en verdad no hay ninguna razón para limitarse a alguns autores(as).

O.I.L.Z.: Teun, esta pregunta está orienta desde el punto de vista metodológico de la investigación crítica. Para realizar un estudio discursivo en $A D$ y ACD se hace fundamental tener un corpus definido; así, para ciertos 
investigadores(as) basta con tener algunas (pocas) muestras discursivas, argumentando con ello la posibilidad de una mayor profundización en el estudio; otros, por su parte, consideran que es necesario compilar y construir un conjunto extenso y ordenado de datos y textos, para que los hallazgos de la descripción y el análisis posean mayores niveles de confiabilidad. ¿Cuál es tu perspectiva al respecto?

T.A.v.D.: Como siempre, esto depende de los objetivos de la investigación. Si quieres comparar cómo la prensa colombiana escribe sobre los indígenas con la manera en que lo hace la prensa venezolana, obviamente no puedes hacerlo solamente sobre la base de una docena de artículos. Muchas veces un estudio más amplio de corpus facilita perfectamente un análisis detallado de una selección de textos típicos. Por supuesto, si uno quiere demostrar algunas ideas nuevas, basta ilustrarlas con algunos ejemplos, como lo he hecho con la noción de manipulación. Pero cuando los objetivos son más empíricos, muchas veces se necesita un estudio de corpus. Para las revistas que dirijo, en general recomendamos trabajar con un corpus, sobre todo para garantizar que el artículo se base en muchos análisis detallados, lo que permite escribir un texto más completo y profundo de un proyecto, y no un artículo ad hoc.

O.I.L.Z.: Una de las orientaciones marcadas del ACD es analizar los discursos de las élites y los grupos hegemónicos dominantes; de igual manera, uno de los principios éticos y axiológicos de esta perspectiva crítica de análisis es solidarizarse con los oprimidos. Es por ello que el ACD debería orientar sus análisis no sólo a los discursos de los grupos privilegiados que abusan del poder, sino también a los de los grupos dominados, es decir, estudiar sus discursos y hacerlos visibles, analizar la manera en que muchas de estas enunciaciones se empoderan y resisten contra los sistemas de dominación y control; desde esta perspectiva, los analistas críticos del discurso contribuirían de manera activa en este proceso de empoderamiento y liberación, ¿Cuál es tu perspectiva al respecto?

T.A.v.D.: Estoy totalmente de acuerdo, y me doy cuenta que en mi caso prefiero estudiar críticamente los discursos de dominación para analizarlos y denunciarlos, inspirados por los demás discursos de los disidentes. Por ejemplo, mis definiciones del racismo se inspiran en las experiencias cotidianas de las minorías o inmigrantes y sus organizaciones. Puede ser que no analizo los discursos mismos de la resistencia, es decir, de los grupos dominados, porque solamente puedo estar de acuerdo. Por ejemplo, leo en el periódico que gran 
parte de los EEUU, y sobre todo los blancos, se escandalizan sobre los contactos de Obama con su reverendo, porque éste ha dicho que EEUU es un país racista y otras cosas del género. Bueno, hasta ahora lo que he visto del reverendo es que, en mi opinión, solamente dice verdades monumentales, que han sido comprobadas en muchos estudios, y que son las experiencias de mucha gente afroamericana y latina en EEUU. Aparentemente a muchas personas no les gusta enfrentarse con esa realidad negativa de su país. Tengo las mismas experiencias en Holanda. El racismo, como el sexismo y el clasismo, se niegan o se ignoran en los discursos dominantes, sobre todo las versiones más cotidianas que están en todas las instituciones, enfocando sobre las versiones más radicales del racismo de la extrema derecha, que representa una parte mucho menos importante y apenas dominante en las Américas o Europa.

O.I.L.Z.: En la mayoría de las perspectivas del ACD se presenta la bipolaridad: Dominante-Dominado; así, existe un grupo privilegiado (Dominante) que enuncia una serie de discursos legitimados a nivel social: docentes, médicos, abogados, religiosos, políticos, entre otros. De igual forma, un grupo dominado al que se controla, domina y abusa a través de estos discursos. Los mismos análisis se pueden bipolarizar también. No obstante Teun, ¿Al analizar los discursos de los grupos hegemónicos y al hacer públicos los hallazgos, no se estaría en últimas contribuyendo a que estos grupos hagan más conscientes y efectivas sus estrategias discursivas, cognitivas y sociales de control, abuso de poder y dominación?

T.A.v.D.: Eso es un tema que hemos comentado mucho en otras ocasiones, y obviamente hay posibilidades de abuso de nuestros trabajos. Pero por otro lado, si después de una lectura de nuestros trabajos los periodistas, políticos y otros, se muestran mucho menos abiertamente racistas en la cobertura, creo que hemos ganado. Es un primer paso importante, porque según algunas teorías psicológicas de la auto-percepción, cuando las personas por presión social se comportan de una manera menos racista, se adaptan también sus actitudes para garantizar la coherencia entre ideas y conducta. Por eso también la importancia de leyes, prescripciones, y otras medidas oficiales, y los buenos ejemplos de las élites, porque ésto genera un consenso que determina que algunas conductas ya no se toleran. Lo anterior también lo hemos visto con la lucha contra el machismo y el sexismo, y las prácticas cotidianas de la discriminación. Obviamente estos cambios no son muy rápidos, y se basan sobre ideologías de siglos, pero con las nuevas generaciones se ve un cambio en una dirección de más igualdad, de más justicia. Nada más basta con comparar 
las actitudes actuales en el Sur de los EEUU (por ejemplo, sobre un candidato negro en las elecciones), con las actitudes dominantes hace solamente 50 años. Lo mismo, por cierto, en América Latina para las actitudes en Colombia y Brasil, entre otros países, sobre la gente de descendencia africana, ésto gracias, sobre todo, a la resistencia de los movimientos afrolatinos.

O.I.L.Z.: Si bien, la Escuela es representada desde los discursos oficiales, educativos, económicos y políticos como un escenario cuyo fin primordial es la formación de individuos analíticos, reflexivos y críticos - ideal de un concepto de educación radical, libertaria y antiautoritaria -, es sabido que constituye un sistema o aparato reproductor de la ideología dominante que busca, en su fin último y velado, la formación de un sujeto productivo, más que productor activo y crítico, que se adhiera con facilidad al sistema socioeconómico y político, manteniendo con ello el status quo, la desigualdad y el "orden social”. Ante esto, ¿Cómo lograr que las instituciones educativas sean verdaderas agentes de transformación social, orientadoras de un pensamiento crítico y libertario, si están sujetas a las políticas dominantes del sistema hegemónico? ¿Cómo resistir ante estos discursos e ideologías, que se naturalizan cada vez más en los docentes y estudiantes, si somos maestros(as) insertos en el sistema de poder?

T.A.v.D.: Tu formulación analítica muestra que conoces el problema, y por tanto, también las dificultades de una solución rápida y fácil. En el momento ya estaría satisfecho si pudiéramos formar a los profesores(as) (y a los profesores(as) de los profesores(as)) en una Universidad verdaderamente crítica. Con todas las formas de desigualdad y de dominación, digo yo, siempre hay que empezar en la propia casa. Es decir, necesitamos programas universitarios profundamente conscientes de las injusticias y desigualdades fundamentales en el mundo, con una enseñanza que no sólo analiza críticamente sino que también muestra cómo se puede actuar en forma concreta. Obviamente, la mayoría de las universidades y disciplinas universitarias no tienen nada que ver con eso. Una vez que tenemos esto en orden, o por lo menos mucho mejor, en nuestra casa, podemos formar profesores y profesoras mejor preparados para la tarea inmensamente difícil de transformar estos aspectos en lo curricular, en los libros de texto y en trabajos de aula, que de verdad son relevantes para los alumnos(as). Durante esa formación, ya pueden aprender a analizar críticamente los libros de texto. Sin esa formación y preparación otros cambios serán casi imposibles. Por ejemplo, hace una década en Chile hubo una iniciativa del Ministerio de Educación de introducir libros de texto 
alternativos para la escuela secundaria pública. Yo conozco esos textos, y son muy interesantes, con análisis crítico de textos, posiciones feministas, pacifistas, etc. Costaban solamente unos dólares y eran mucho mejores que los libros de texto de las (caras) escuelas públicas. Pero para poder usar estos libros, se necesitaba profesores bien formados, y la idea fue formarlos(as) en cursos masivos por el país entero. Por desgracia, éstos y otros problemas hicieron que el gobierno abandonara el proyecto casi revolucionario; también porque la mayoría del profesorado no estaba preparado para enseñar con libros de texto que apenas comprendían ellos(as) mismos(as). Es cierto que los cambios hay que empezarlos a todos los niveles, y no podemos esperar hasta que se cambie la universidad (y sus viejos profesores) y los programas universitarios de educación del profesorado, pero obviamente la clave del cambio está allá.

O.I.L.Z.: Es muy importante lo que planteas, se hace necesario y fundamental, casi un imperativo, que las universidades formen para el desarrollo del pensamiento complejo y crítico; no obstante, por lo menos en la mayoría universidades de mi región, prima un discurso acrítico, y afirmativo de las políticas estatales dominantes, que como ya afirmé, buscan la adhesión del individuo al sistema productivo. Ante esto Teun, ¿En que podría contribuir el $A C D$, como perspectiva crítica de análisis, a la formación de una Universidad libertaria...?

T.A.v.D.: Primero, un análisis (auto) crítico de los programas, los materiales de las clases y de los discursos en el aula. Ésto apenas se hace, y me parece fundamental en la formación de los(las) estudiantes, y para la transformación de la universidad. Pero con buenas estrategias. Simplemente atacando a los/las profesores(as) se puede tener el efecto opuesto. Esos trabajos tienen que persuadir y seducir el profesorado, mostrando, por ejemplo, que los cambios también son en sus propios intereses. Segundo, introducir y mostrar contenidos, métodos, principios alternativos. Invitar a los docentes en otros departamentos o universidades que sí siguen una línea alternativa de enseñanza.

O.I.L.Z.: En la entrevista que hicimos el año pasado, y que fue publicada en la Revista Latinoamericana de Estudios del Discurso, de la ALED (Venezuela), mencionaste que acababas de terminar un libro extenso sobre teoría del contexto. ¿Qué se puede encontrar en tu nuevo trabajo?

T.A.v.D.: En la actualidad estoy corrigiendo las pruebas del primero de los dos libros nuevos sobre contexto, Discourse and Context, que será publicado por Cambridge University Press, con una traducción española publicada por 
Gedisa, como la mayoría de mis libros. Un poco después viene otro libro sobre contexto con Cambridge U.P. cuyo título todavía no sabemos, también será publicado en versión española por Gedisa.

O.I.L.Z.: Teniendo en cuenta que el conocimiento no es estático sino que está en constante transformación, base fundamental para el desarrollo del pensamiento y la problematización del conocimiento, quiero preguntarte si has o no reformulado en la actualidad algunos conceptos o planteamientos de tus anteriores libros, por ejemplo: "La ciencia del texto", "Texto y contexto", "Las estructuras y funciones del discurso". "La noticia como discurso. Comprensión, estructura y producción de la información”, entre otros.

T.A.v.D.: Por supuesto, porque estoy cambiando permanentemente. Resumir esos cambios sería cuestión de muchas horas o páginas de entrevista, pero en un par de comentarios cortos puedo decir primero que los trabajos de los últimos 25 años son mucho más críticos, sociales y políticos. Por eso el movimiento hacía el ACD. Temas como el racismo y la ideología obviamente son muy diferentes de mis viejos temas como coherencia, macroestructuras semánticas, procesos mentales o la estructura de la noticia. Segundo, la investigación se ha hecho mucho más multidisciplinaria. Mis libros sobre ideología y sobre contexto son trabajos que integran la lingüística, la filosofía y casi todas las disciplinas sociales. Tercero, al lado de seguir con estudios críticos concretos, por ejemplo sobre racismo en España y América Latina, me gustan también los trabajos sobre los fundamentos de nuestra disciplina, como en el proyecto sobre discurso y contexto, y ahora en el nuevo proyecto sobre discurso y conocimiento.

O.I.L.Z.: Desde el 2007 editas la nueva revista virtual sobre Estudios del Discurso, Discurso \& Sociedad (www.dissoc.org), que se ha constituido en uno de los espacios más importantes para la publicación de artículos, entrevistas y reseñas de libros, entre otros géneros en la web. ¿Cómo surge la idea de crear esta revista? ¿Qué recepción ha tenido en los ciberlectores?

T.A.v.D.: La recepción de DISCURSO \& SOCIEDAD ha sido muy buena, creo poder deducir ésto de las visitas frecuentes a la página. Tenemos más de 20.000 accesos por mes, más de 2.000 visitas de más de 2.000 lectores(as) que bajan más de 10.000 archivos cada mes. Obviamente, ésto es mucho más que cualquier revista académica de papel. La revista anterior Discurso y Sociedad (con "y" y no con "\&"), que era muy buena, por desgracia no podía continuar, 
también por problemas de distribución. La revista digital es gratis, y llega a todos los(as) profesores(as) y especialmente a los(as) estudiantes. Porque no solamente publicamos textos originales de España y de América Latina sino también traducciones de textos sobre todo de la hermana revista mayor DISCOURSE \& SOCIETY. La revista se ha hecho un recurso muy importante, ya después de un año, y espero que con los años pueda consolidarse como una revista de referencia sobre los estudios del discurso, junto con las demás que existen, como la Revista Latinoamericana de Estudios del Discurso de la ALED, y la revista y página de nuestros amigos(as) en Buenos Aires (www. discurso.org). La idea fue precisamente eso, primero continuar Discurso y Sociedad de una manera gratis y para todos(as), y tener una revista hermana iberoamericana de la revista DISCOURSE \& SOCIETY para quienes prefieren publicar en español. Con ALED como organización, espero también que DISCURSO \& SOCIEDAD sea un recurso fundamental para un espacio autónomo de estudios del discurso. Ya es la región, creo yo, con más interés en estudios del discurso en el mundo, y la única zona con su propia organización profesional. Obviamente, para reforzar la revista, su prestigio, y finalmente su indexación internacional, los criterios de los artículos tienen que ser rigurosos. En ocasiones llegan artículos que de ninguna manera satisfacen los criterios internacionales en estudios del discurso, y obviamente con más oferta, vamos a ser cada vez más selectivos. Básicamente, la gente puede tomar DISCOURSE \& SOCIETY como el criterio de calidad, porque son artículos que han sido evaluados no solamente por mí, primero, sino después por los mejores especialistas del mundo en un área. Por tanto, publicamos no más de un $20 \%$ de los artículos que nos envían. Espero para DISCURSO \& SOCIEDAD tener los mejores trabajos de la gente joven de America Latina que hacen sus tesis de maestría y doctorado; de igual manera, los mejores trabajos del resto del mundo, así como todos los trabajos excelentes que nos envían en los últimos años de China, Hong Kong, Taiwán y Corea. Recomiendo a la gente joven, primero, aprender o mejorar su inglés para poder seguir los debates y publicaciones internacionales, y segundo, participar activamente en los desarrollos más interesantes en los estudios del discurso en el globo entero, y no solamente en Europa.

O.I.L.Z.: Sin duda Teun, es satisfactorio poder encontrar un espacio en la web con la calidad de DISCURSO \& SOCIEDAD, y dejo en claro, no obstante, que también existen otras publicaciones virtuales importantes, es decir, serias en sus ediciones y objetivos. Poder acceder a una publicación de manera gratuita facilita los procesos de reconceptualización y actualización de los(las) académicos(as). Es sabido que en muchas ocasiones la distribución 
y adquisición de las revistas o libros de AD y ACD es complicada, por varios motivos: algunas revistas no llegan a las universidades de provincia, anexo a ésto se requiere ser, en ocasiones, miembro de las asociaciones de estudios del discurso para recibir ciertas publicaciones, las editoriales que distribuyen y comercializan los textos de AD y ACD no tienen sucursales en los municipios pequeños, lo que hace complicada la adquisición... Algunos textos no se encuentran... entre otros. Es por ello que DISCURSO \& SOCIEDAD se erige como una publicación mucho más cercana... mucho más accesible...

Ante lo expuesto anteriormente Teun, en ocasiones hago una reflexión y quiero compartirla contigo: Algunas veces, y te lo digo por la experiencia en mi universidad, son pocos(as) docentes y estudiantes universitarios que abordan el $A D$ y el $A C D$, sobre todo, como perspectiva crítica de análisis en las regiones pequeñas; sin embargo, ya a nivel nacional, en los centros urbanos mayores, se amplía el espectro de investigación crítica... Pareciera que esta distribución académica e investigativa jerarquizara y determinara los desarrollos del $A D$ y el ACD en el país, puesto que se constituyen en una referencia inmediata y casi que obligada, debido a que en las regiones mayores tienen más acceso a los adelantos y teorías de base: publicaciones, grupos de investigación, postgrados en la línea... latinoamericanos y europeos. Con ello se estaría conformando una élite de analistas críticos, que regulan los procesos de adquisición del conocimiento e investigación. ¿Cuál es tu perspectiva al respecto?

T.A.v.D.: La división entre centro y periferia se manifiesta de todos lados, en las universidades, el país, la región y en el mundo, y tanto económicamente como académicamente. Y no sólo es una cuestión de marginalización o no, sino también, como dices, una jerarquización: Los del centro tienen más recursos, y también por eso son mejores, también porque pueden contratar los mejores investigadores del país. Por desgracia esta situación es tan compleja que casi no se puede hacer nada, a menos que los gobiernos inviertan más recursos a las universidades de la provincia. Pero ocurre aquí lo que se dice en la Biblia: será dado a quienes ya tienen. Una estrategia de romper un poco esta forma de centralización es el uso de Internet. Si los(las) académicos(as) de las universidades de la provincia tienen un computador, y los de cualquier universidad en general, pueden tener acceso directo a los trabajos no solamente en el centro del país, sino también en el mundo. Mi página está abierta para todos y todas, y se usa mucho precisamente por estudiantes y profesores(as) que no están en el centro. 
O.I.L.Z.: Finalmente Teun, ¿Qué proyectos estás desarrollando en la actualidad? ¿Cuáles son tus intereses teóricos e investigativos actuales? ¿Cuándo volverás a Colombia?

T.A.v.D.: Como mencioné, finalmente puedo continuar con un proyecto que empecé hace seis años pero que quedó atrasado por el trabajo sobre contexto que pensaba poder terminar en un año, pero que duró más de cinco, y ese es mi proyecto sobre discurso y conocimiento. Dos nociones fundamentales de las ciencias humanas y sociales. También será un proyecto multidisciplinario, con aportes de la lingüística, la epistemología, la psicología y la Inteligencia Artificial, la antropología y la sociología, ciencias y disciplinas que han trabajado mucho tiempo sobre conocimiento. Pero lo extraño es que a pesar de esto no hay libros sobre discurso y conocimiento. Hay muchos estudios que tocan un aspecto, como del gran elefante, los temas conocidos sobre el tópico y el comentario, focus, presuposición, pronombres, "Common Ground", y las estrategias epistémicas de la distribución de la información en las oraciones, temas que quiero expandir al discurso, combinándolos con lo que se sabe en psicología sobre el manejo del conocimiento en la producción y la comprensión, y la psicología social de las cogniciones sociales y compartidas, con la sociología del conocimiento, etc., y así todo en un marco multidisciplinario complejo. Todavía no sé que corpus voy a usar, pero creo que me gustaría volver al estudio de las noticias, porque las noticias en los medios de comunicación constituyen los discursos de los que todo el mundo aprende más, e influyen en la construcción de sus conocimientos sobre el mundo. Vale la pena hacer un libro sobre el rol de la producción del conocimiento con las noticias. Este es mi trabajo. En la Pompeu Fabra esperamos empezar con un proyecto multidisciplinar sobre estrategias epistémicas con quince investigadores(as), cada un(a) estudiando un género, como libros de texto, interrogatorios, programas de televisión, blogs de ciencias en Internet, etc. Ya comprenderás que todo esto va a ser un trabajo de muchos años. Este año se publican muchos de mis libros (originales o en traducción - ve la lista de mis publicaciones en mi página de Internet www.discursos.org), pero en los años que siguen quiero desarrollar primero varias investigaciones antes de publicar libros nuevos. ¡Gracias por tus preguntas interesantes!

O.I.L.Z.: ;Gracias a ti por tus respuestas interesantes!, Teun. 